EPJ manuscript No.

(will be inserted by the editor)

\title{
Oscillatory behavior and enhancement of the surface plasmon linewidth in embedded noble metal nanoparticles
}

\author{
Rafael A. Molina, Dietmar Weinmann, and Rodolfo A. Jalabert \\ Institut de Physique et Chimie des Matériaux de Strasbourg, UMR 7504, CNRS-ULP \\ 23 rue du Loess, 67037 Strasbourg Cedex, France
}

September 6, 2002

\begin{abstract}
We study the Landau damping of the surface plasmon resonance of metallic nanoparticles embedded in different environments of experimental relevance. Important oscillations of the plasmon linewidth as a function of the radius of the nanoparticles are obtained from numerical calculations based on the time dependent local density approximation. These size-oscillations are understood, within a semiclassical approximation, as a consequence of correlations in the spectral density of the nanoparticles. We treat inert matrices, as well as the case with an unoccupied conduction band. In the latter case, the plasmon lifetime is greatly reduced with respect to the inert case, but the non-monotonous size-dependence persists.
\end{abstract}

PACS. 71.45.Gm Exchange, correlation, dielectric and magnetic response functions, plasmons - 31.15.Gy Semiclassical methods $-36.40 . \mathrm{Vz}$ Optical properties of clusters

\section{Introduction}

In recent years, the use of femtosecond laser pulses has made it possible to investigate the time evolution of collective and quasi-particle excitations in metallic nanoparticles. In these experiments, the actual time-resolved evolution of the energy transfer to the environment can be studied [1, 2, 3]. A determining factor in the relaxation processes is the lifetime of the collective excitations (surface plasmons), which for small cluster sizes $(0.5 \mathrm{~nm} \lesssim R \lesssim$ $2 \mathrm{~nm}$ ), is limited by Landau damping (decay into electronhole pairs). The lifetime of the surface plasmon resonance is thus, not only a problem of fundamental interest, but also important for possible applications.

In the last decade, the analogy of surface plasmons with nuclear giant resonances has been exploited, in order to obtain a physical understanding, as well as analytical and numerical estimations, of the plasmon lifetime [4, 6, 6, [7]. Following these approaches, and using a semiclassical formalism, an oscillatory behavior of the level width was determined for free clusters of small sizes (a number of atoms $N$ between $N=20$ and $N=1000$ ). These analytical results have been verified against numerical calculations within the time-dependent local density (TDLDA) for a jellium model [8], and the oscillations in the level width with the size of the nanoparticles were shown to arise from the electron-hole density-density correlations in the angular restricted density of states.

The non-monotonous size-dependence of the plasmon lifetime has been experimentally observed in free alkaline metal nanoparticles [9, 10], as well as in embedded noble metal clusters [11]. Further experimental and theoretical work would be needed in order to unambiguously characterize the level width oscillations. While the analytical calculations are most easily done for free alkaline clusters, metallic noble metal clusters embedded in a transparent matrix are more conveniently investigated from the experimental point of view [2]. It is then important to study theoretically the size-dependence of plasmon lifetime for embedded particles. This is the purpose of this work, where we extend the results of Ref. [7] to different kinds of environments.

The problem of the plasmon lifetime in metallic nanoparticles embedded in a matrix, is considerably more difficult, and less understood, than in the case of free clusters. The most obvious effect when the clusters are embedded in a dielectric material, is the change produced by the dielectric constant of the matrix, that moves the position of the plasmon resonance according to the Mie formula [13], and can also affect the width of the plasmon [7]. Moreover, when the matrix is not inert, it can react with the atoms in the surface of the nanoparticle. The electronic states on the surface are modified and contribute to the loss of coherence of the collective state. This is the so-called chemical interface damping. In addition, the presence of a conduction band in the matrix at an energy accessible to the electrons, weakens the confinement, and contributes to the width of the surface plasmon in an important way. All these different effects can add up and make the theoretical description of the system quite involved.

The experiments of Charlé et al. [11] studied the influence of the matrix environments on surface plasmon excitations of small silver particles. They found a strong broadening of the surface plasmon resonance when the 
particles were embedded in a reactive matrix such as CO, as compared to an inert matrix from a noble gas like Ar. This effect has been attributed to chemical interface damping. Also, a very strong broadening has been observed by Hövel et al. 114 for silver particles embedded in $\mathrm{SiO}_{2}$. In a very interesting and pioneering paper, Persson proposed a model for explaining these experimental observations that took into account the modification of the electronic eigenstates of the metallic nanoparticle by the layer of absorbates on its surface and by a conduction band [15].

In the next section we recall the basic theoretical facts of the calculation of the plasmon lifetime within a semiclassical approximation. We then study the linewidth of embedded clusters from TDLDA calculations for inert matrices. The case of matrices with a conduction band is treated in Sec. 4. The theoretical treatment of the chemical interface damping is out of reach of our theoretical description, but the cases that we will consider are not thought to be considerably influenced by this effect.

\section{Theoretical background for free clusters}

The Landau damping of the dipole plasmon can be calculated treating the collective excitation as an external perturbation, which can give rise to the creation of electronhole excitations [12]. Then, Fermi's golden rule yields the linewidth

$$
\Delta \Gamma(R)=2 \pi \sum_{p h}|\langle p|\delta V| h\rangle|^{2} \delta\left(\hbar \omega_{\mathrm{M}}-\epsilon_{p}+\epsilon_{h}\right),
$$

where $|p\rangle$ and $|h\rangle$ are electron and hole states in the selfconsistent field, with its energies given by $\epsilon_{p}$ and $\epsilon_{h}$, respectively, $\delta V$ is the dipole field due to the surface plasmon, and $\omega_{\mathrm{M}}$ is the frequency of the plasmon, which classically is given by the Mie formula,

$$
\omega_{\mathrm{M}}=\frac{\omega_{\mathrm{p}}}{\sqrt{\epsilon_{d}+2 \epsilon_{m}}}
$$

where $\omega_{\mathrm{p}}$ is the plasma frequency, $\epsilon_{d}$ is the dielectric function of the $d$ electrons and $\epsilon_{m}$ is the dielectric function of the embedding matrix

In the case of spherical symmetry, assuming that the confinement and the interactions lead to hard walls at radius $R$ in the self-consistent field, we can evaluate Eq. (11). Integrating over the electron-hole states, one obtains 5

$$
\begin{aligned}
& \Delta \Gamma(R)=c \gamma \int_{E_{\mathrm{F}}}^{E_{\mathrm{F}}+\hbar \omega_{\mathrm{M}}} \mathrm{d} E \sum_{L} \sum_{L^{\prime}=L \pm 1}(2 L+1)\left(2 L^{\prime}+1\right) \\
& \times<L, 0 ; 1,0 \mid L^{\prime}, 0>^{2} E\left(E-\hbar \omega_{\mathrm{M}}\right) d_{L}(E) d_{L^{\prime}}\left(E-\hbar \omega_{\mathrm{M}}\right)
\end{aligned}
$$

where $\left\langle L, 0 ; 1,0 \mid L^{\prime}, 0\right\rangle$ is a Clebsch-Gordan coefficient, $\gamma=\left(2 \pi \hbar^{3}\right) /\left(3 N M^{2} \omega_{\mathrm{M}} R^{4}\right), c=4 M R^{2} / \hbar^{2}, E_{\mathrm{F}}$ is the Fermi energy, and $d_{L}(E)$ is the one-dimensional density of states with total angular momentum $L$.
Using the semiclassical expression for the density of states of the one-dimensional problem [16], we can decompose $d_{L}(E)$ in its smooth (zero-length trajectories) and oscillating components (arising from periodic orbits). We then obtain two contributions to the width of the plasmon resonance [7]

$$
\Delta \Gamma=\Gamma_{0}+\Gamma_{\mathrm{osc}} .
$$

The smooth term $\Gamma_{0}$ arises from the smooth component of $d_{\mathrm{L}}$ and exhibits the well known $1 / R$ dependence firstly proposed by Kawabata and Kubo 17,5

$$
\Gamma_{0}(R)=\frac{3 \hbar}{4} \frac{v_{\mathrm{F}}}{R} g(\xi)
$$

where $\xi=\hbar \omega_{\mathrm{M}} / E_{\mathrm{F}}, g(\xi)$ is a smoothly decreasing function with $g(0)=1$, and $v_{\mathrm{F}}$ is the Fermi velocity.

The oscillating part $\Gamma_{\mathrm{osc}}$ arises from the density oscillations as a function of the energy. Within a semiclassical approach, it can be written as [7]

$$
\Gamma_{\mathrm{osc}} \approx \frac{6 \sqrt{2 \pi} \hbar}{M R^{2} \sqrt{k_{\mathrm{F}} R \xi^{3}}} \sum_{r=1}^{\infty} \frac{1}{\sqrt{r}} \cos \left(2 r k_{\mathrm{F}} R \xi\right),
$$

where the sum runs over all repetitions $r$ of the period of the equivalent one-dimensional motion. The amplitude of this oscillations can be of the order of $\Gamma_{0}$ for small clusters.

\section{Embedded metallic clusters in inert matrices}

We want to address the question of what happens with the oscillations and the typical value of the linewidth of the plasmon when the nanoparticles are embedded in a matrix. In Ref. [7] we presented some calculations for noble metals embedded in inert matrices. The numerical results were obtained using the TDLDA formulation by Bertsch [18 but modifying the residual interaction to include the frequency-dependent dielectric function of the $d$ electrons $\epsilon_{d}(\omega)$ and the dielectric constant of the matrix $\epsilon_{m}$ [19]. In order to maintain the spherical symmetry of our problem we always considered cluster sizes corresponding to magic numbers of atoms.

For inert matrices, the plasmon linewidth is affected only by the modification of the frequency $\omega_{M}$ through the parameter $\xi$ of Eqs. (5) and (6). This is due to the fact that the self-consistent potential, and, therefore, the electronhole density-density correlation function are practically unchanged by $\epsilon_{m}$, but the energy position of the plasmon peak follows the Mie formula. Results for the width of the plasmon in the case of Ag nanoparticles embedded in a matrix of Ar are shown in Fig. 1. In the inset we show a typical spectrum of the photo-absorption cross-section for $\mathrm{Ag}_{440}$ embedded in Ar. The singularities of the spectrum are smeared out by a non-zero $\gamma$. This value of $\gamma$ is subtracted at the end of the calculation and we verify that the fit of $\Delta \Gamma$ is not sensitive to it, provided that it is larger than a minimum value. $\Delta \Gamma$ exhibits pronounced oscillations as a function of the radius. The smooth part of 


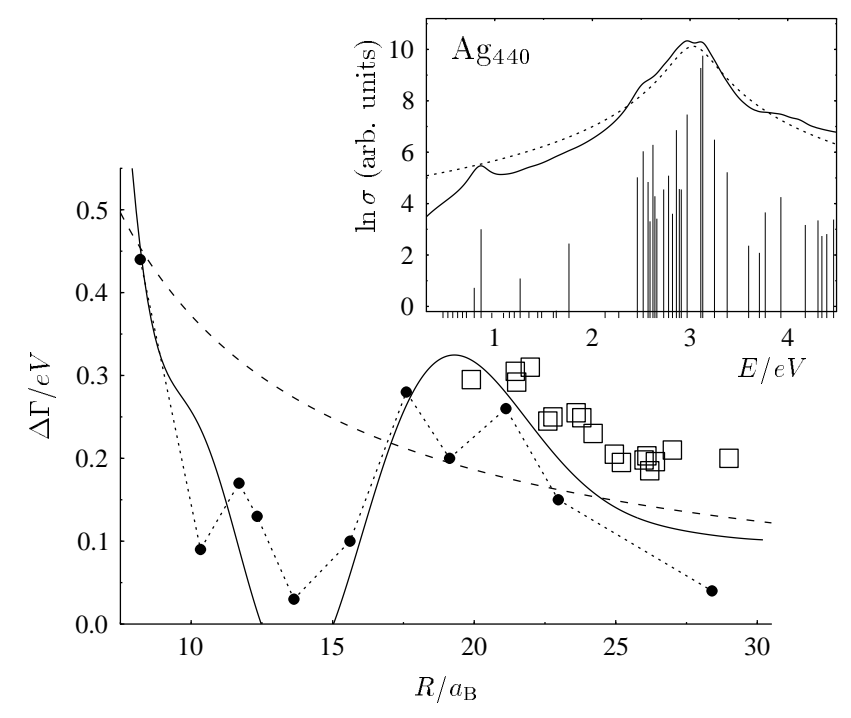

Fig. 1. Linewidth as a function of the radius (in units of the Bohr radius $a_{\mathrm{B}}=0.53 \AA$ ) for silver nanoparticles in an $\mathrm{Ar}$ matrix calculated within TDLDA (full circles) together with the experimental results of Ref. [11] (empty squares). The dotted line through the numerical points is a guide-to-the-eye. The dashed and the solid lines represent, respectively, $\Gamma_{0}(R)$ and $\Delta \Gamma(R)$ according to Eqs. (田), (5) and (6) (with a reduction factor of 3 as discussed in the text). Inset: logarithm of the TDLDA absorption cross section for $\operatorname{Ag}_{440}\left(R / a_{\mathrm{B}}=2.2\right)$, showing the pronounced surface plasmon resonance, fitted by a Lorentzian (dotted line). The excited states are indicated by tick marks and their oscillation strengths given by the height of the vertical lines.

the linewidth, and our semiclassical result, are an overall factor of 3 larger than the numerical results. Therefore, they have been rescaled for in the figure. The experimental values of Charlé et al. (empty squares) are relatively well described by the TDLDA results (within a $20 \%$ ). The oscillations are suppressed due to the large particle sizes and the smearing resulting from wide size-distributions. The difference between the Kubo formula, on one hand, and the numerical and experimental results, on the other hand, is not understood. In any case, given the approximations used in the semiclassical calculation, it is not surprising that, although, this simple theory works well with alkaline metals, when we include $\epsilon_{d}(\omega)$ there are factors not taken into account. Nevertheless, the analytically obtained oscillations have the correct period and relative size.

Moving through different values of $\epsilon_{m}$ we change the position of the plasmon frequency $\omega_{M}$. Since the electronhole density-density correlation function is insensitive to $\epsilon_{m}$, the plasmon lifetime behaves in a non-monotonous fashion as a function of $\epsilon_{m}$. Thus, we can use the dielectric constant of the embedding medium as a probe for this density-density correlation function. As an example, we show in Fig. 2 the results of TDLDA calculations for the width of the surface plasmon resonance for silver clusters of two different sizes $(N=92$ and $N=198)$ as a

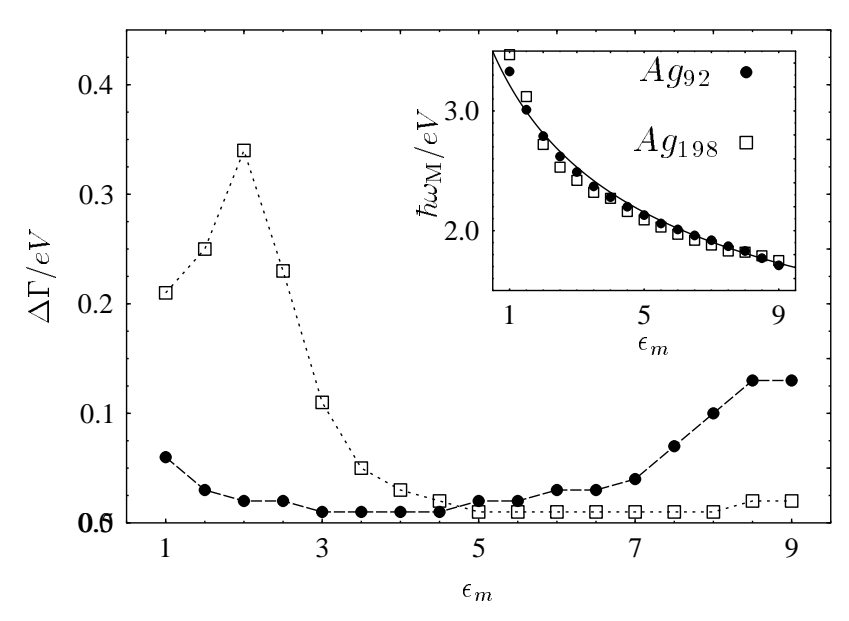

Fig. 2. Width of the surface plasmon resonance for silver clusters of two different sizes as a function of $\epsilon_{m}$. Inset: frequency of the plasmon $\omega_{M}$ together with the expression for the Mie formula (solid line) with a small modification of the bulk $\omega_{\mathrm{p}}$.

function of $\epsilon_{m}$. The oscillations in the width are very different for the two sizes due to the shell filling and the corresponding density correlation function. Although the range of $\epsilon_{m}$ over which we can see the complete oscillation of the plasmon lifetime is very large, it should be possible to observe maxima and minima in experiments, if we are able to change the dielectric constant of the surrounding medium. In the inset of this figure we show the position of the plasmon peak as a function of the $\epsilon_{m}$ for the corresponding sizes. The behavior with $\epsilon_{m}$ is very well described by the Mie formula, and the two sizes behave in the same way. We can see almost no difference in the position of the plasmon peak between the sizes for the same value of $\epsilon_{m}$, but there are great changes in its width.

\section{$4 \mathrm{Ag}$ nanoparticles embedded in a matrix with a conduction band}

The $\mathrm{SiO}_{2}$ used in experiments for embedding $\mathrm{Ag}$ nanoparticles is an amorphous solid with a conduction band with a minimum situated at $-1.7 \mathrm{eV}$ with respect to the vacuum energy. The valence band maximum occurs $10.6 \mathrm{eV}$ below the vacuum energy and has no influence in the Ag-surface plasmon [15]. Chemical interaction in the surface between $\mathrm{Ag}$ and the $\mathrm{SiO}_{2}$ is not expected to occur 14 and chemical interface damping does not influence the width of the plasmon in this case. In order to implement the TDLDA calculations, we simulate the embedded medium by a change in the boundary conditions for the calculation in the selfconsistent potential $V(r)$ so that $V(r) \rightarrow-1.7 \mathrm{eV}$ when $r \rightarrow \infty$. In this way the electrons are less bounded to the cluster and can go to the conduction band in the matrix. The results of the self-consistent calculation of $V(r)$ and its comparison with the potential without conduction band are shown in the inset of Fig. 3. We can clearly see 
that the electrons at $E_{\mathrm{F}}$ are less tight, which translates in a small redshift in the position of the plasmon peak due to the bigger spill-out and into an increase (by a factor of 2) of the width (compare with Fig. 1). This is consistent with the factor of 3 enhancement observed in the experiments of Ref. 14. Although in these experiments the distribution of sizes has certain unknown variance that can increase the results for the linewidth of the optical absorption experiments, a more refined model including the self-interaction correction to the TDLDA, and a corrected dielectric function for the surface, are probably needed for a more quantitative agreement. However, we can clearly see that the non-monotonous behavior is maintained and is of the same order as for free Ag nanoparticles. Other different kinds of glasses that are used in experiments should produce similar increases in the width of the plasmon if the difference between the energy of the minimum of the conduction band and $E_{\mathrm{F}}$ is not too far from the energy of the plasmon.

\section{Conclusions}

As other size-dependent phenomena in clusters, the width of the plasmon presents a leading-order (smooth) contribution (that goes like $1 / R$ ), with oscillatory corrections due to shell effects. For the plasmon lifetime these sizedependent corrections are much more important than for the position of the resonance. Going for free to embedded clusters makes this difference still more pronounced.

We have shown in this work that the size-dependent oscillations of the linewidth, in free as well as in embedded clusters, arise from electron-hole density correlations. Such an effect can be explored by varying the dielectric constant of the matrix around the nanoparticle.

We have also considered the width of the plasmon for a simple model of nanoparticles of $\mathrm{Ag}$ surrounded by a matrix of amorphous $\mathrm{SiO}_{2}$ with a conduction band. The width is increased in a way that agrees semi-quantitatively with the experiments. The oscillations in the width are still present and could be seen in experiments with a narrow distribution of sizes. An adequate choice of the composition of the matrix should make it possible to increase the lifetime of the surface plasmon by tuning its energy to a value where the electron-hole correlation function is nearly zero, which can be useful in certain applications.

We thank J.-Y. Bigot, H. Haberland, and P.A. Hervieux for helpful discussions. RAM acknowledges the financial support from the European Union's Human Potential Program (contract HPRN-CT-2000-00144).

\section{References}

1. J.-Y. Bigot, J.-C. Merle, O. Crégut, A. Daunois, Phys. Rev. Lett. 75, 4702 (1995).

2. V. Halté, J. Guille, J.-C. Merle, J. I. Perakis, J.-Y. Bigot, Phys. Rev. B 60, 11738 (1999); J.-Y. Bigot, V. Halté J.-C. Merle, A. Daunois, Chem. Phys. 251, 181 (2000).

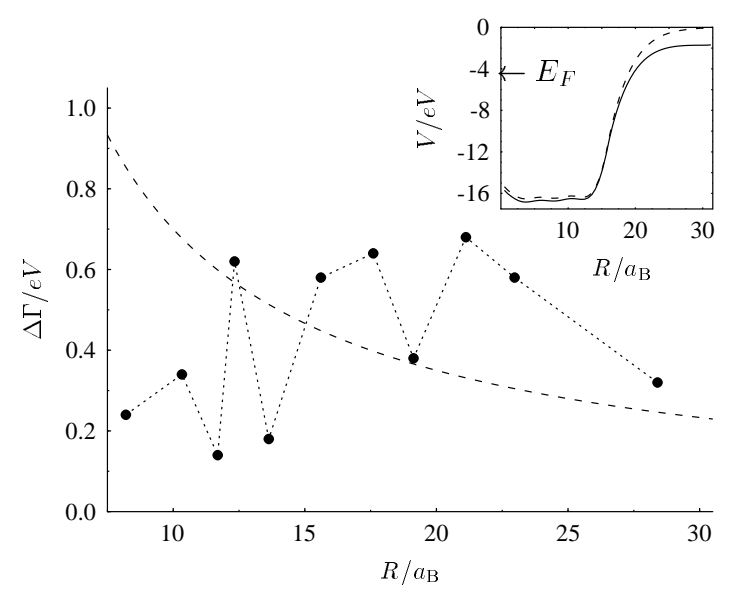

Fig. 3. Width of the surface plasmon resonance as a function of the radius of the particle for silver clusters embedded in $\mathrm{SiO}_{2}$ (the matrix is assumed to support a conduction band, see text). The discontinuous line is the best fit to $1 / R$ behavior. Inset: comparison of the self-consistent potential $V(r)$ with (full line) and without (dashed line) the conduction band. We indicate the position of the Fermi energy with an arrow.

3. T. Klar, M. Perner, S. Grosse, G. von Plessen, W. Spirkl, J. Feldmann, Phys. Rev. Lett. 80, 4249 (1998).

4. M. Barma, V. Subrahmanyam, J. Phys.: Condens. Matter 1, 7681 (1989).

5. C. Yannouleas and R.A. Broglia, Ann. Phys. (N.Y.) 217, 105 (1992).

6. C. Yannouleas, E. Vigezzi, R.A. Broglia, Phys. Rev. B 47, 9849 (1993); C. Yannouleas, Phys. Rev. B 58, 6748 (1998).

7. R.A. Molina, D. Weinmann and R.A. Jalabert, Phys. Rev. B 65, 155427 (2002).

8. W. Ekardt, Phys. Rev. B 31, 6360 (1985).

9. C. Bréchignac, Ph. Cahuzac, J. Leygnier, A. Sarfati, Z. Phys. D 12, 2036 (1993).

10. S. Mochizuki, M. Sasaki, R. Ruppin, J. Phys.: Condens. Matter 9, 5801 (1997).

11. K.P. Charlé, W. Schulze, B. Winter, Z. Phys. D 12, 471 (1989).

12. Oscillations in Finite Quantum Systems, by G. F. Bertsch and R. A. Broglia, Cambridge University Press, Cambridge, England (1994).

13. Optical properties of Metal Clusters, by U. Kreibig and M. Vollmer, Springer, Berlin (1995).

14. H. Hövel, S. Fritz, A. Hilger, U. Kreibig, and M. Vollmer, Phys. Rev. B 48, 18178 (1993).

15. B. N. J. Persson, Surf. Sci. 281, 153 (1993).

16. Chaos in Classical and Quantum Mechanics by M.C. Gutwiller, Springer-Verlag, Berlin (1990).

17. A. Kawabata, R. Kubo J. Phys. Soc. Jpn. 21, 1765 (1966).

18. G. Bertsch, Comp. Phys. Comm. 60, 247 (1990).

19. A. Rubio and L. Serra, Phys. Rev. B 48, 9028 (1993). 